\title{
Isolated Bladder Endometriosis in a Patient With Previous Cesarean Sections
}

\author{
Chye Lee Kho Lilya, c, Charissa Goh ${ }^{\mathrm{a}}$, Yong Kuei Lim ${ }^{\mathrm{b}}$
}

\begin{abstract}
Urological endometriosis is rare and accounts for approximately $1 \%$ of women with endometriosis. The pathophysiology of bladder endometriosis may be divided into two different causes, namely primary and secondary. The primary form occurs spontaneously and manifests in a form of a generalized pelvic disease whereas the secondary form is thought to be iatrogenic and typically occurs after pelvic surgery such as Cesarean section or hysterectomy. We present a case report on a patient presenting with an invasive bladder mass initially thought to be from a pelvic malignancy. The final histology showed isolated bladder endometriosis. She had a significant past medical history of two previous Cesarean sections. A review of her magnetic resonance imaging (MRI) images demonstrated the presence of a Cesarean scar niche which may be linked to her bladder endometriosis. There is a need to improve awareness of the possible link between Cesarean scar niche with bladder endometriosis. This is important as it raises the clinical question of whether appropriate repair of Cesarean scar niches can prevent future complications such as bladder endometriosis which can potentially be associated with significant morbidity.
\end{abstract}

Keywords: Bladder endometriosis; Cesarean scar niche; Rare cases

\section{Introduction}

Endometriosis is defined as endometrial glands and stroma at extrauterine sites. These ectopic implants most commonly involve the ovaries, ovarian fossa, uterosacral ligaments, rectovaginal septum, and the pouch of Douglas. While endometriosis is common and can be found in up to $10 \%$ of women of reproductive age [1], urinary tract involvement is rare and

Manuscript submitted July 5, 2020, accepted September 9, 2020

Published online September 23, 2020

aDivision of Obstetrics and Gynaecology, KK Women's and Children's Hospital, Singapore

${ }^{\text {b} T i m o t h y ~ L i m ~ C l i n i c ~ f o r ~ W o m e n ~ a n d ~ C a n c e r ~ S u r g e r y, ~ M o u n t ~ A l v e r n i a ~ H o s-~}$ pital, Singapore

${ }^{\mathrm{c} C}$ Corresponding Author: Chye Lee Kho Lily, Division of Obstetrics and Gynaecology, KK Women's and Children's Hospital, 229899 Singapore.

Email: kho.chye.lee@singhealth.com.sg

doi: https://doi.org/10.14740/jmc3543 accounts for approximately $1 \%$ of women with endometriosis [2].

The etiology of endometriosis of the urinary tract is not entirely clear. General theories of endometriosis include retrograde menstruation, celomic metaplasia, lymphatic or hematogenous dissemination of endometrial cells, spread of endometrium-derived stem or progenitor cells, and altered genetic or immune factors [3]. The pathophysiology of bladder endometriosis may be divided into two different causes, namely primary and secondary. The primary form occurs spontaneously and manifests in a form of a generalized pelvic disease whereas the secondary form is thought to be iatrogenic and typically occurs after pelvic surgery such as Cesarean section or hysterectomy [4].

We present a case report on a patient presenting with an invasive bladder mass initially thought to be from a pelvic malignancy. The final histology showed isolated bladder endometriosis. She had a significant past medical history of two previous Cesarean sections.

\section{Case Report}

In November 2017, a 49-year-old lady, para 2, was referred to our gynecology unit for further evaluation of an incidental left ovarian mass with bladder infiltration found on a health screen. She presented with symptoms of a 1-year history of increased urinary frequency every $2 \mathrm{~h}$ without any urinary incontinence. This was not associated with any abdominal pain, nocturia, dysuria or hematuria. Her menses were infrequent and she experienced spotting every 6 months due to her Mirena, which she had changed every 5 years since 2003. She had a past medical history of a laparoscopic myomectomy in 1998 and two previous Cesarean sections in 2001 and 2003 respectively. She was up to date with her cervical smears and mammograms and they were unremarkable. She had a significant family history of ovarian cancer wherein her mother was diagnosed with ovarian cancer with metastases to the colon at the age of 75 .

On examination, her abdomen was soft, non-tender and there were no obvious palpable masses. A lower transverse incision was noted. Speculum examination revealed a normal cervix. A left adnexal mass measuring $4 \mathrm{~cm}$ was palpable bimanually.

The ultrasound pelvis revealed an enlarged left ovary which was solid with heterogeneous echogenicity. There was no hypervascularity noted. No other adnexal masses or free 

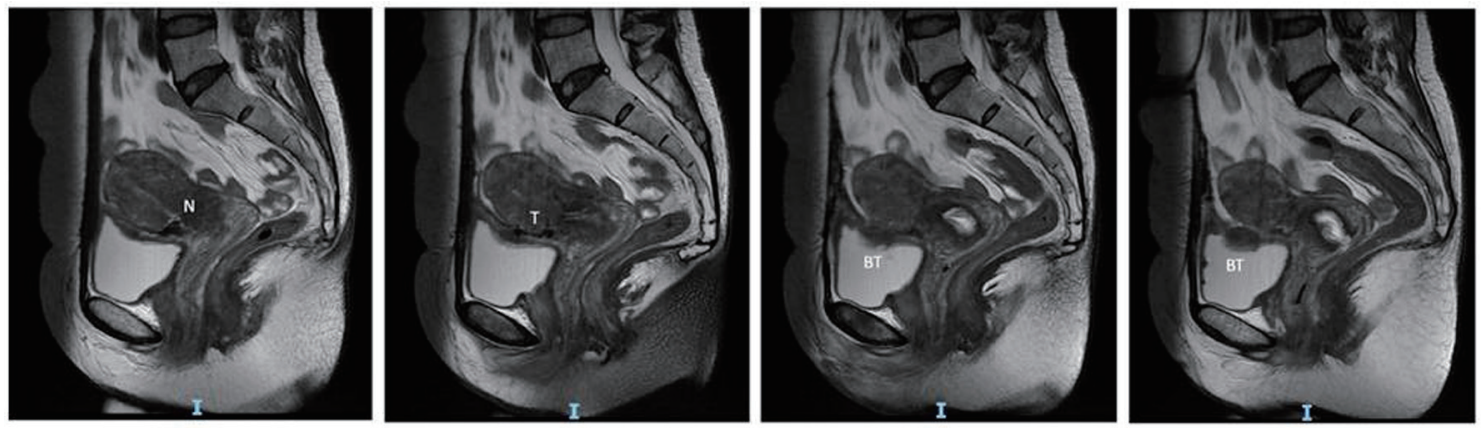

Figure 1. MRI images showing the sagittal view of the Cesarean scar niche $(\mathrm{N})$, tract $(\mathrm{T})$ and bladder tumor $(\mathrm{BT})$. MRI: magnetic resonance imaging.

fluid was noted. A 4-cm irregular papillary mucosal lesion was noted at the left lateral wall of the urinary bladder posteriorly and this was medial to the left ureteric orifice. The lesion appeared cystic and the posterior wall of the bladder appeared to be infiltrated. Normal ureteric jet was observed. An ultrasound of the kidneys confirmed normal sized kidneys bilaterally.

Her ovarian tumor markers were normal. The cancer antigen-125 (CA125) was $8 \mathrm{U} / \mathrm{mL}, \mathrm{CA} 19-9$ was $7 \mathrm{U} / \mathrm{mL}$, alphafetoprotein was $2.4 \mathrm{ng} / \mathrm{mL}$ and carcino-embryonic antigen was $1.2 \mathrm{ng} / \mathrm{mL}$. Her creatinine was normal at $58 \mu \mathrm{mol} / \mathrm{L}$.

The computed tomography (CT) scan of her thorax, abdomen and pelvis showed a left ovarian mass measuring $4.6 \times$ $4.2 \times 2.9 \mathrm{~cm}$, suggestive of a left ovarian tumor. The inferior border of the ovarian mass was noted to be inseparable from the adjacent uterus. The ovarian mass was also noted to be contiguous with a small focus of irregular soft tissue thickening involving the superior left side of the bladder, measuring about $1.2 \times 0.4 \mathrm{~cm}$. No lymphadenopathy or metastasis was identified.

A magnetic resonance imaging (MRI) of the pelvis was ordered to further characterize the ovarian mass. A $4.1 \times 3.7 \times$ $4.9 \mathrm{~cm}$ enhancing mass was noted to be inseparable from the left side of the uterus and was deemed to be likely uterine in origin (Fig. 1). Bilateral ovaries were small and the left ovary appeared to be separate from the mass. An area of nodular thickening and enhancement of the superior wall of the bladder measuring about $2.5 \mathrm{~cm}$ in diameter was noted. It exhibited T1 hyperintense and $\mathrm{T} 2$ hypointense foci. No lymphadenopathy or ascites was seen.

She was counselled for midline laparotomy, total abdominal hysterectomy, bilateral salpingectomy keep in view oophorectomy, frozen section keep in view staging and partial bladder cystectomy with bilateral double J-stent insertion with the urologist on standby. A cystoscopy and bilateral double Jstent insertion was performed prior to the surgery. Cystoscopy revealed a tumor plaque in the bladder dome measuring approximately $4 \mathrm{~cm}$, suspicious for invasion from an endometrial mass (Fig. 2). Bilateral ureteric orifices were normal. Bilateral double J-stents were subsequently inserted. A midline incision was performed. On inspection of the pelvis, a 5-cm left uterine fundal fibroid and normal ovaries bilaterally were noted. Intraperitoneal survey was normal. Figure 3 is the intraoperative image showing an endometrial mass with invasion into the bladder. The decision was made for a partial cystectomy with resection of the mass in the bladder. En-bloc resection of the bladder mass and total hysterectomy bilateral salpingectomy was performed with conservation of the ovaries. Figure 4 shows the resected specimen sent for histology. Frozen section of the bladder mucosa revealed endometriosis and cystitis glandularis. The patient had an unremarkable recovery and was discharged home well on the fourth post-operative day with an indwelling urinary catheter. A micturating cystourethrogram was performed 2 weeks after the surgery that showed no evidence of contrast leak. The urinary catheter was removed successfully. She was subsequently followed up in the gynecology clinic with regular scans for surveillance of the ovaries.

The final histology showed endometriosis involving the bladder mucosa. A benign leiomyoma was also noted. There were also no findings of concurrent endometriosis or adenomyosis in the specimens sent.

\section{Discussion}

The most common site of urological endometriosis is the bladder. A previous case series by Donnez et al found that 4 out of 17 patients had previous Cesarean sections [5]. Endometriotic lesions of the bladder are closely located to the uterus and typically affect the detrusor muscle in the bladder trigone and bladder apex [6].

Most patients with bladder endometriosis present with cyclical suprapubic and back pain with irritative voiding symptoms worsened during menstruation. Approximately $20 \%$ of patients would experience cyclical menstrual hematuria, a pathognomonic sign for bladder endometriosis [6]. Patients can also present with symptoms suggestive of pelvic endometriosis such as a dysmenorrhea and dyspareunia [6]. Our patient presented in an atypical manner and did not complain of any symptoms suggestive of pelvic or bladder endometriosis. In a study by Vercellini et al [7], only 2 out of the 40 women who had bladder endometriosis were asymptomatic. We postulate that the absence of menstrual symptoms suggestive of pelvic endometriosis may be possibly related to the use of Mirena.

In the assessment of urological endometriosis, both cystoscopy and MRI have been shown to be useful modalities. Cystoscopy has also been noted to be useful in the evalua- 

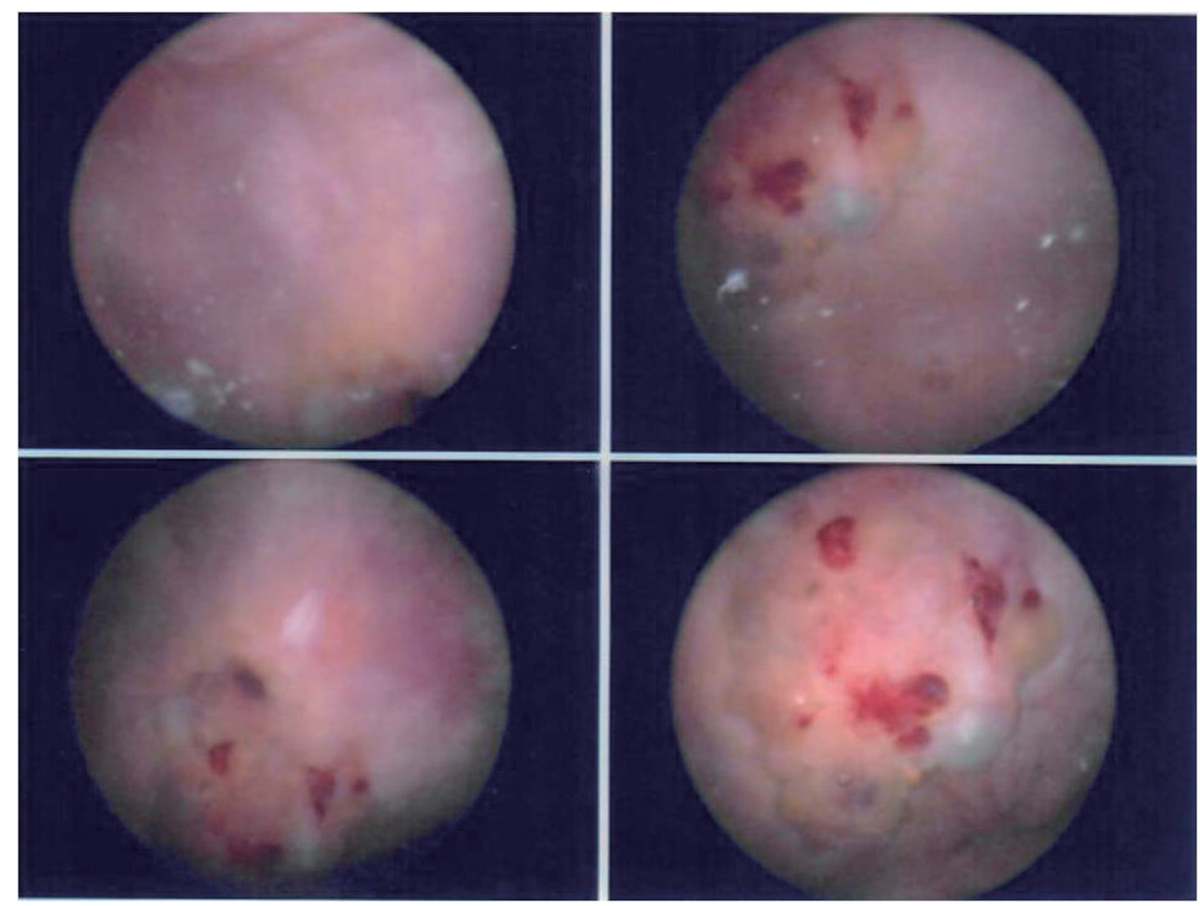

Figure 2. Cystoscopy images showing a suspicious tumor with involvement of the bladder mucosa.

tion of bladder endometriosis as concurrent biopsies can be performed to confirm endometriosis. Bladder endometriomas have a typical appearance of edematous bluish submucosal multilocular lesions in the bladder dome or bladder base on cystoscopy [6]. These findings were similar to the intraoperative cystoscopy findings in our patient.

MRI has been shown to have an accuracy of up to $98 \%$ in diagnosis of endometriosis [6]. MRI is also beneficial in assessing the extent and severity of endometriosis in other sites [8]. In our patient, the diagnosis was not clear before surgery as the patient did not have typical features of endometriosis. Her initial pelvic ultrasound and CT scan were suspicious for

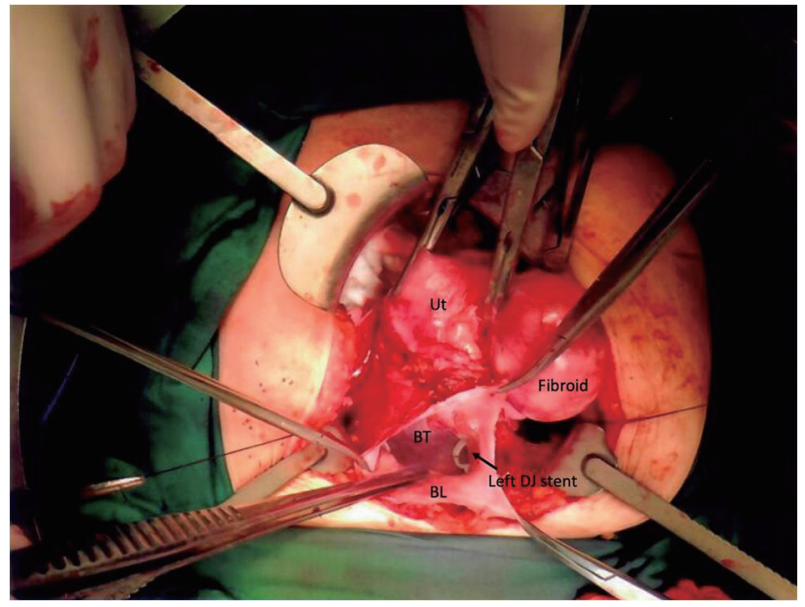

Figure 3. Intraoperative image showing the relationship between the bladder (BL), bladder tumor (BT), uterus (Ut) and fibroid. The bladder tumor was inseparable from the uterus. a possible pelvic malignancy with bladder invasion. Moreover, she did not have clinical features of endometriosis as she was asymptomatic and her CA125 was not elevated. The MRI showed features of possible bladder endometriosis such as T1 hyperintense and T2 hypointense foci in the bladder lesion. However, as malignancy could not be entirely excluded, surgery was performed for histological diagnosis.

In our retrospective review of the MRI findings of our patient with hindsight of the final histology, we noted that the area of bladder endometriosis was closely associated with the Cesarean scar niche and tract. This suggests that the isolated bladder endometriosis in our patient may be secondary to her previous Cesarean section and Cesarean scar niche. Our theory

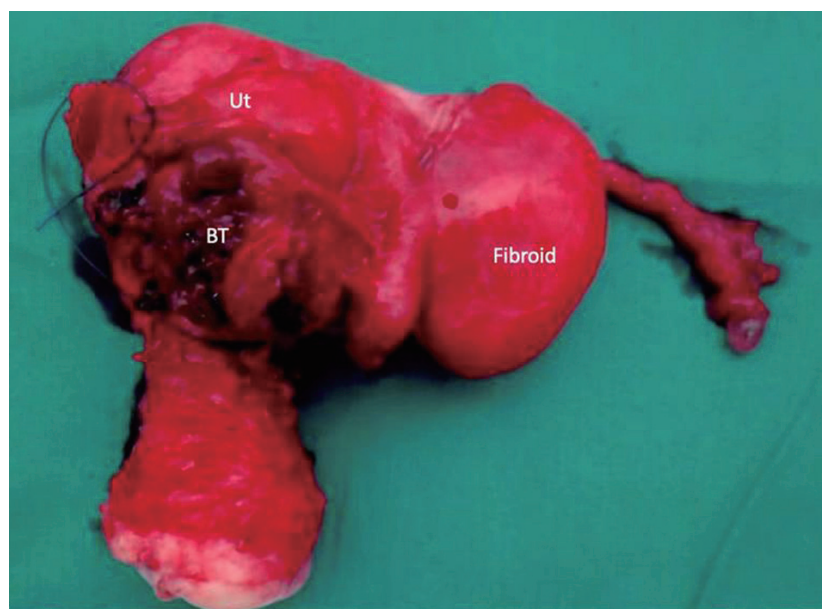

Figure 4. Resected specimen consisting of the bladder, bladder tumor (BT), uterus (Ut), bilateral tubes and fibroid. 
is also supported by the fact that our patient only had isolated bladder endometriosis with no endometriosis seen elsewhere in the pelvis both macroscopically and microscopically. The patient also has no recurrence of endometriosis 3 years postoperatively despite conservation of her ovaries. A similar case of isolated bladder endometriosis in a patient with two prior Cesarean sections has been described by Ayari et al [9]. The patient underwent transurethral resection of bladder tumor and was started on estrogen-progesterone medications postoperatively. At her 3-month follow-up, there were no signs of recurrence. Another previous case report by Posner et al [10] described a patient with bladder endometriosis and two prior Cesarean sections who underwent bilateral salpingo-oophorectomy, hysterectomy, partial cystectomy, distal right ureterectomy, and ureteroneocystostomy. During pelvic exploration, a solitary endometrioma was noted involving the distal right ureter, right posterior bladder wall and uterus. As the mass was mobilized from the uterus, an attenuated myometrium was encountered, which appeared to be the site of a prior Cesarean section. Histologic examination of the surgical specimen confirmed the diagnosis of vesical endometriosis that extended from the uterus to the bladder mucosa. No other endometriomas were identified. These findings correlate to the MRI findings in our patient. However, it is difficult to say if the tract seen on MRI is the cause of her bladder endometriosis or a result of chronic endometriosis. There is also no prior data on Cesarean scar niche and the occurrence of bladder endometriosis, and it is also not known if repair of the niche can prevent such a complication. Moving forward, more attention should be paid to the Cesarean scar niche to establish if there is a correlation between the niche and endometriosis although this would be challenging because of the rarity of the condition.

\section{Conclusions}

Bladder endometriosis can often mimic pelvic malignancy resulting in radical surgeries. They can be a rare complication of a previous Cesarean section. More data is needed to determine the relationship of Cesarean scar niche in bladder endometriosis.

\section{Acknowledgments}

None to declare.

\section{Financial Disclosure}

None to declare.

\section{Conflict of Interest}

None to declare.

\section{Informed Consent}

It was obtained from the patient.

\section{Author Contributions}

CLKL summarized the case, performed the literature review, and wrote the manuscript; $\mathrm{CG}$ performed the literature review and wrote the manuscript; YKL supervised the write up of case and was the surgeon in charge of the care of the patient.

\section{Data Availability}

The authors declare that data supporting the findings of this study are available within the article.

\section{References}

1. Shafrir AL, Farland LV, Shah DK, Harris HR, Kvaskoff M, Zondervan K, Missmer SA. Risk for and consequences of endometriosis: A critical epidemiologic review. Best Pract Res Clin Obstet Gynaecol. 2018;51:1-15.

2. Berlanda N, Vercellini P, Carmignani L, Aimi G, Amicarelli F, Fedele L. Ureteral and vesical endometriosis. Two different clinical entities sharing the same pathogenesis. Obstet Gynecol Surv. 2009;64(12):830-842.

3. Sourial S, Tempest N, Hapangama DK. Theories on the pathogenesis of endometriosis. Int J Reprod Med. 2014;2014:179515.

4. Mettler L, Gaikwad V, Riebe B, Schollmeyer T. Bladder endometriosis: possibility of treatment by laparoscopy. JSLS. 2008;12(2):162-165.

5. Donnez J, Spada F, Squifflet J, Nisolle M. Bladder endometriosis must be considered as bladder adenomyosis. Fertil Steril. 2000;74(6):1175-1181.

6. Charatsi D, Koukoura O, Ntavela IG, Chintziou F, Gkorila $\mathrm{G}$, Tsagkoulis $\mathrm{M}$, Mikos T, et al. Gastrointestinal and urinary tract endometriosis: a review on the commonest locations of extrapelvic endometriosis. Adv Med. 2018;2018:3461209.

7. Vercellini P, Frontino G, Pisacreta A, De Giorgi O, Cattaneo M, Crosignani PG. The pathogenesis of bladder detrusor endometriosis. Am J Obstet Gynecol. 2002;187(3):538-542.

8. Beaty SD, Silva AC, De Petris G. Bladder endometriosis: ultrasound and MRI findings. Radiol Case Rep. 2006;1(3):92-95.

9. Ayari Y, Boussaffa H, Sellami A, Zaghbib S, Sami BR, Nouira Y. An isolated bladder endometriosis misdiagnosed as a bladder tumor. Urol Case Rep. 2020;28:101062.

10. Posner MP, Fowler JE, Jr., Meeks GR. Vesical endometriosis 12 years after a cesarean section. Urology. 1994;44(2):285-287. 